\title{
Interventions to reduce emergency department consultation time: A systematic review of the literature
}

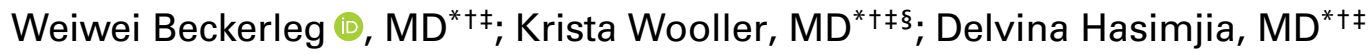

\author{
CLINICIAN'S CAPSULE \\ What is known about the topic? \\ Prolonged consultation to decision time and ED length of \\ stay are associated with adverse outcomes. \\ What did the study ask? \\ What interventions have been tried to reduce consultation \\ to decision time and, in turn, ED length of stay? \\ What did the study find? \\ A variety of interventions have been found to be effective \\ in reducing consultation to decision time and ED length \\ of stay. \\ Why does this study matter to clinicians? \\ Findings from this review can help inform quality \\ improvement efforts in streamlining ED flow.
}

\section{ABSTRACT}

Objectives: Overcrowding in the emergency department (ED) is associated with increased morbidity and mortality. Studies have shown that consultation to decision time, defined as the time when a consultation has been accepted by a specialty service to the time when disposition decision is made, is one important contributor to the overall length of stay in the ED.

The primary objective of this review is to evaluate the impact of workflow interventions on consultation to decision time and ED length of stay in patients referred to consultant services in teaching centres, and to identify barriers to reducing consultation to decision time.

Methods: This systematic review was performed in accordance with the PRISMA guidelines. An electronic search was conducted to identify relevant studies from MEDLINE, EMBASE, Cochrane Central, and CINAHL databases. Study screening, data extraction, and quality assessment were carried out by two independent reviewers.

Results: A total of nine full text articles were included in the review. All studies reported a decrease in consultation to decision time post intervention, and two studies reported cost savings. Interventions studied included short messaging service
(SMS) messaging, education with audit and feedback, standardization of the admission process, implementation of institutional guideline, modification of the consultation process, and staffing schedules. Overall study quality was fair to poor. Conclusions: The limited evidence suggests that audit and feedback in the form of SMS messaging, direct consultation to senior physicians, and standardization of the admission process may be the most effective and feasible interventions. Additional high-quality studies are required to explore sustainable interventions aimed at reducing consultation to decision time.

\section{RÉSUMÉ}

Contexte: L'encombrement des services des urgences (SU) est associé à une augmentation de la morbidité et de la mortalité. Des études ont démontré que le temps écoulé entre les demandes de consultation et les prises de décision, défini comme le temps passé entre le moment où une demande de consultation en spécialité est acceptée et la prise de décision quant aux suites à donner, joue un rôle important dans le prolongement de la durée totale de séjour au SU.

Objectifs: L'étude avait pour objectifs principaux d'évaluer l'incidence des interventions de rationalisation du travail sur le temps écoulé entre les demandes de consultation et les prises de décision et sur la durée de séjour au SU chez les patients dirigés vers des services de consultation dans des centres hospitaliers d'enseignement, et de cerner les obstacles à la réduction de ce temps d'attente.

Méthode: II s'agit d'une revue systématique réalisée conformément aux lignes directrices PRISMA. Une recherche électronique a été menée dans les bases de données MEDLINE, EMBASE, Cochrane Central et CINAHL afin de relever les études pertinentes. Deux examinateurs ont procédé, chacun de leur côté, à la sélection des études, à l'extraction des données et à l'évaluation de la qualité des études retenues. Résultats: Ont été sélectionnés, au total, 9 articles en version intégrale. Dans toutes les études, on faisait état, après les interventions, d'une diminution du temps écoulé entre les demandes de consultation et les prises de décision et, dans

From the *The Ottawa Hospital, Ottawa, ON; †Faculty of Medicine, University of Ottawa, Ottawa, ON; ¥Department of Medicine, The Ottawa Hospital; and the §Ottawa Hospital Research Institute, Ottawa, ON.

Correspondence to: Dr. Krista R. Wooller, Division of General Internal Medicine, D107c Civic Campus Box 209, 1053 Carling Avenue, Ottawa, ON K1Y 4E9; Email: krwooller@toh.ca

(C) Canadian Association of Emergency Physicians

CJEM 2020;22(1):56-64

DOI 10.1017/cem.2019.435 
deux études, d'une économie de coûts. Parmi les interventions examinées, il y avait les services de messages courts (SMS), la formation du personnel suivie de contrôles et de commentaires, l'uniformisation du processus d'admission, la mise en œuvre de lignes directrices dans les établissements, la modification du processus de consultation et les horaires de travail du personnel. Dans l'ensemble, la qualité des études variait de passable à piètre.

Conclusions: Malgré le petit nombre de données probantes, il semblerait que les contrôles et les commentaires sous forme de SMS, les demandes de consultation envoyées directement aux médecins principaux et l'uniformisation du processus $d^{\prime}$ admission soient les interventions les plus efficaces et les plus faciles à mettre en œuvre. II faudrait aussi réaliser des études de qualité sur des interventions durables visant à réduire le temps écoulé entre les demandes de consultation et les prises de décision.

Keywords: Education, quality improvement, research methods, residents and fellows, systematic review

\section{INTRODUCTION}

Emergency department (ED) wait time has been identified as an important quality indicator in our healthcare system. Overcrowding in the ED is associated with increased morbidity and mortality, prolonged inpatient stay, and decreased patient satisfaction. ${ }^{1-4}$ Studies have shown that consultation to decision time, defined as the time when a referral has been accepted by a consultant service to the time when disposition is made, is one component that can contribute to the overall length of stay in the $\mathrm{ED},{ }^{5-7}$ making up $26 \%-67 \%$ of total ED length of stay. ${ }^{8,9}$ Consultations are common, occurring in up to $40 \%$ of all ED visits. ${ }^{10}$ Unique challenges exist at teaching centres to optimize the efficiency of the consultation process due to the presence of trainees, because they often require more time to perform assessments and need to review with more senior staff before a disposition decision is reached. Bernstein et al. found a reduction in ED length of stay when consultations were performed by more senior clinicians. ${ }^{2}$

Given the increasing pressures to improve flow in ED and the important role the consultation process can play in prolonging ED length of stay, it is essential to evaluate interventions designed to streamline the consultation process to determine their impact on reducing consultation to decision time and improving patient outcomes. To our knowledge, no previous systematic review has been conducted on this topic. While Lee et al. published a review of consultations in the ED, their focus was primarily on understanding the proportion of ED patients requiring consultant referral and practices aimed at reducing ED consultations. Only one study on intervention to reduce consultation to decision time was included, and it showed a reduction in consultation time after the Dean of the College of Medicine mandated a policy to improve efficiency. ${ }^{11}$
The objectives of this systematic review were to evaluate the impact of workflow interventions on the consultation to decision time and ED length of stay in patients referred to consultant services in teaching centres, as well as to identify any barriers to reducing consultation to decision time. Association between consultation to decision time and ED length of stay with patient safety outcomes will also be explored.

\section{METHODS}

\section{Protocol and registration}

This systematic review was performed in accordance with the Preferred Reporting Items for Systematic Reviews and Meta-Analyses (PRISMA) guidelines. ${ }^{12}$ No protocol was registered for this review.

\section{Eligibility criteria}

Studies examining single or combined interventions aimed at reducing consultation to decision time in the ED for adult patients ( $\geq 18$ years of age) presenting to the ED and requiring referrals to consultant services were included. A broad criterion was employed for the types of interventions, such as instituting guidelines or targets on consultation to decision time, reorganizing shifts for medical personnel, education, and performance feedback, to be as inclusive as possible. The setting was limited to EDs at teaching centres given the unique challenges present in the consultation process due to the presence of trainees, with no restriction on urban versus rural EDs. Observational studies and randomized controlled trials were considered for inclusion. Studies that did not assess interventions aimed at reducing consultation to decision time were excluded, as were those that 
did not include consultation to decision time in the ED as an outcome measure. Studies were restricted to the English language.

\section{Information sources}

An electronic search was conducted to identify relevant studies from MEDLINE, EMBASE, Cochrane Central, and CINAHL databases, including studies from 1946 to May 2018. A health information specialist was involved in carrying out the search, with input from the research team. The full electronic search strategy can be found in the supplementary materials. Reference lists of all included studies were screened for additional articles that were missed by the initial search.

\section{Study selection}

Firstly, all titles and abstracts were screened by two independent reviewers, with a third reviewer involved to resolve any discrepancies. EndNote was used to manage all citations. Secondly, the full-text of selected articles were reviewed by two independent reviewers, and decision was made regarding inclusion or exclusion based on the previously defined criteria. A third reviewer was involved to resolve any discrepancies when required in order to reach consensus.

\section{Data collection}

Data were subsequently extracted by two independent reviewers using Excel worksheets. Data items included study design, location, population, type of intervention, impact of intervention on consultation to decision time, ED length of stay and patient safety, and barriers to application of intervention.

\section{Risk of bias}

Study quality was rated separately by two reviewers using the SQUIRE 2.0 checklist for quality improvement initiatives as a reference. ${ }^{13}$ This was selected given that other validated scales are better applied to traditional scientific research projects.

The completeness of reporting was assessed by further adapting the SQUIRE ratings using prior literature as a guide. $^{14,15}$

Briefly, the reviewers assessed whether each aspect of the guideline item was reported in the manuscript, as per the 18-item SQUIRE criteria. Studies were scored either Yes (+1) or No (0) for each of the statements, and, when the guideline statements contained multiple descriptors, they were recorded as complete, partially complete, or incomplete based on the number of statements supported by the study. Subsequently, each study was categorized as meeting the "poor/fair/good" criterion based on the total score of the combined descriptors.

\section{Analysis of results}

A descriptive qualitative analysis was conducted in this systematic review. A meta-analysis could not be performed given the heterogeneity in the included studies.

\section{RESULTS}

\section{Study selection}

Abstracts totalling 1,919 were identified through the literature search, with an additional abstract identified from the references of included studies, for a total of 1,920 abstracts, as summarized in the PRISMA flow diagram (Figure 1). A repeat literature search was conducted for troubleshooting, with one additional study identified, because it was not indexed in any of the major databases. The kappa value for inter-rater agreement on the inclusion or exclusion of the titles or abstracts was 0.96 . After the initial screening process, 51 abstracts were included, of which 34 full articles were retrieved for full review. The remainder were conference proceedings or abstracts that were not published as full articles.

Of the full articles retrieved for review, 25 were excluded. Reasons for exclusion were listed in Figure 1. The kappa value for inter-rater agreement on the inclusion or exclusion of full articles was 0.94 .

\section{Study characteristics}

Data were extracted for location, population, types of interventions, study design and quality, outcomes, sustainability, and measures (Table 1).

\section{Study location and population}

All studies were conducted in North America and South Korea within the past decade. Most of them took place at academic centres, with one conducted at a community 


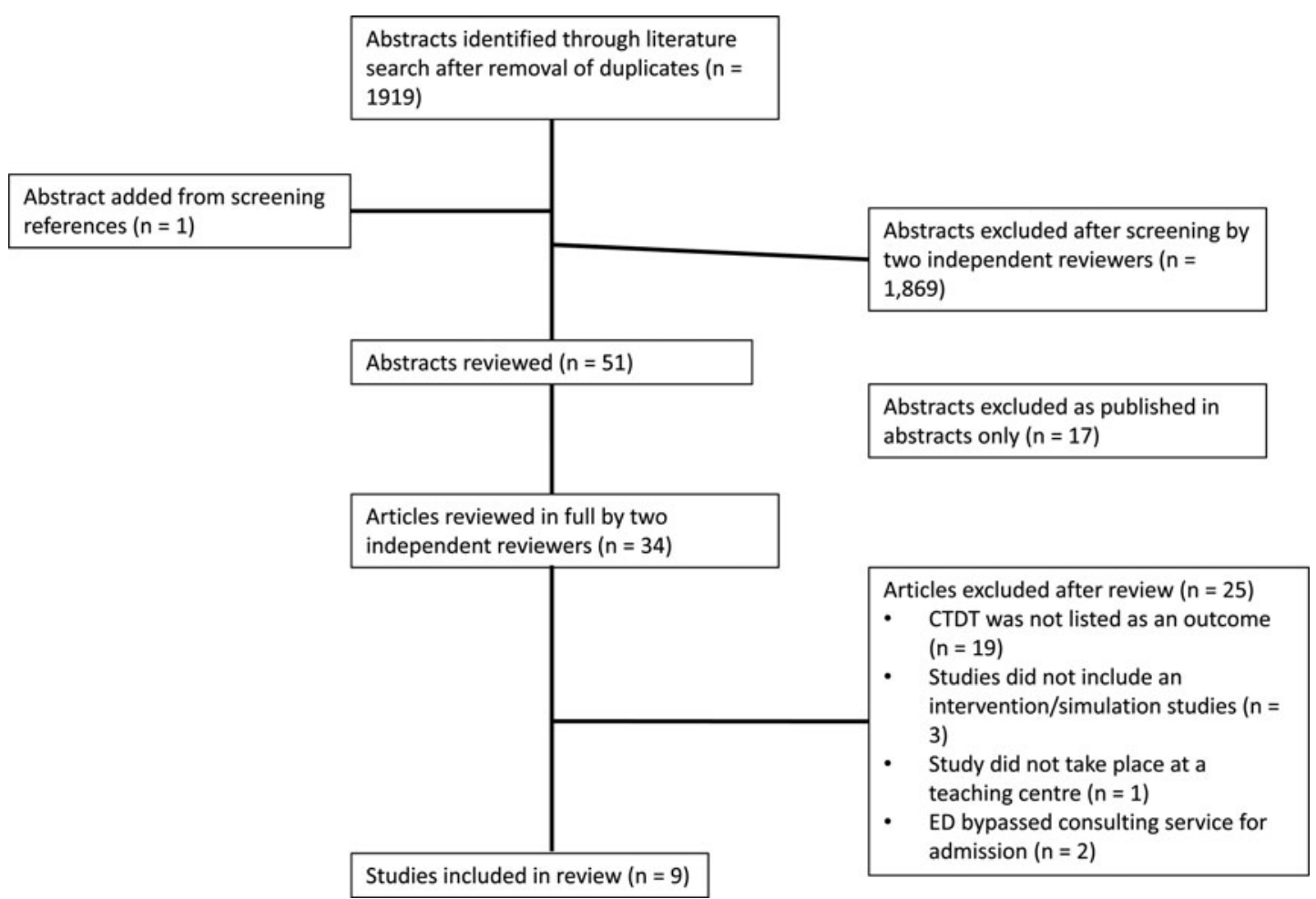

Figure 1. PRISMA flow diagram of study selection process

teaching hospital. ${ }^{23}$ Four studies included patients requiring internal medicine consultation only, ${ }^{1922-24}$ one study population comprised patients requiring psychiatric consultation, ${ }^{21}$ another study included general surgical patients only, ${ }^{17}$ and the remaining three studies incorporated all patients for whom consultation was requested in the ED. ${ }^{16,18,20}$

\section{Interventions, outcomes, and study quality}

Eight of the nine studies included are observational pre and post studies, and one was a retrospective chart review. The kappa value for inter-rater agreement on study quality was 0.77 . Results are summarized based on interventions trialed.

\section{SMS messaging reminder for delays in consultation process}

This strategy was used in the studies published by Cho et al. and Kim et al. ${ }^{16,18}$ Both studies included all patients in the ED who required a consultant referral. In the study by Cho et al., ${ }^{16}$ SMS messaging was sent if a resident from the consulting service has not arrived in the ED 20 minutes after the initial consultation request was submitted electronically. Another SMS message was sent out at 3 hours to all residents in the consulting department, and a final message was sent at 6 hours to all residents and attending physicians in the consulting department if no disposition decision has been made. Consultation to decision time was reduced by $101 \mathrm{~min}-$ utes and ED length of stay by 106 minutes. Outcomes were tracked for 40 days post-intervention. There was no change in time to discharge from the ED after a disposition decision was made, and the proportion of inpatient admissions did not change. The study quality was rated as fair given the limitations of a short monitoring period, lack of data on consultation volume preintervention, and lack of assessment of provider experience.

Similarly, the study done by Kim et al. sent out SMS messages at 2 and 4 hours to the attending physicians in the consultant department, if no disposition has been reached then. ${ }^{18}$ Another SMS was sent at 8 hours to the attending consultants and the admissions office if admitted patients were still boarding in the ED. Consultation to decision time was reduced by 16 minutes and ED length of stay by 14 minutes for consulted patients. Time from admission decision to patients leaving the ED also decreased by 12 minutes. The follow-up period 


\section{Table 1. Summary of study design, population, intervention, outcomes, measures, and study quality}

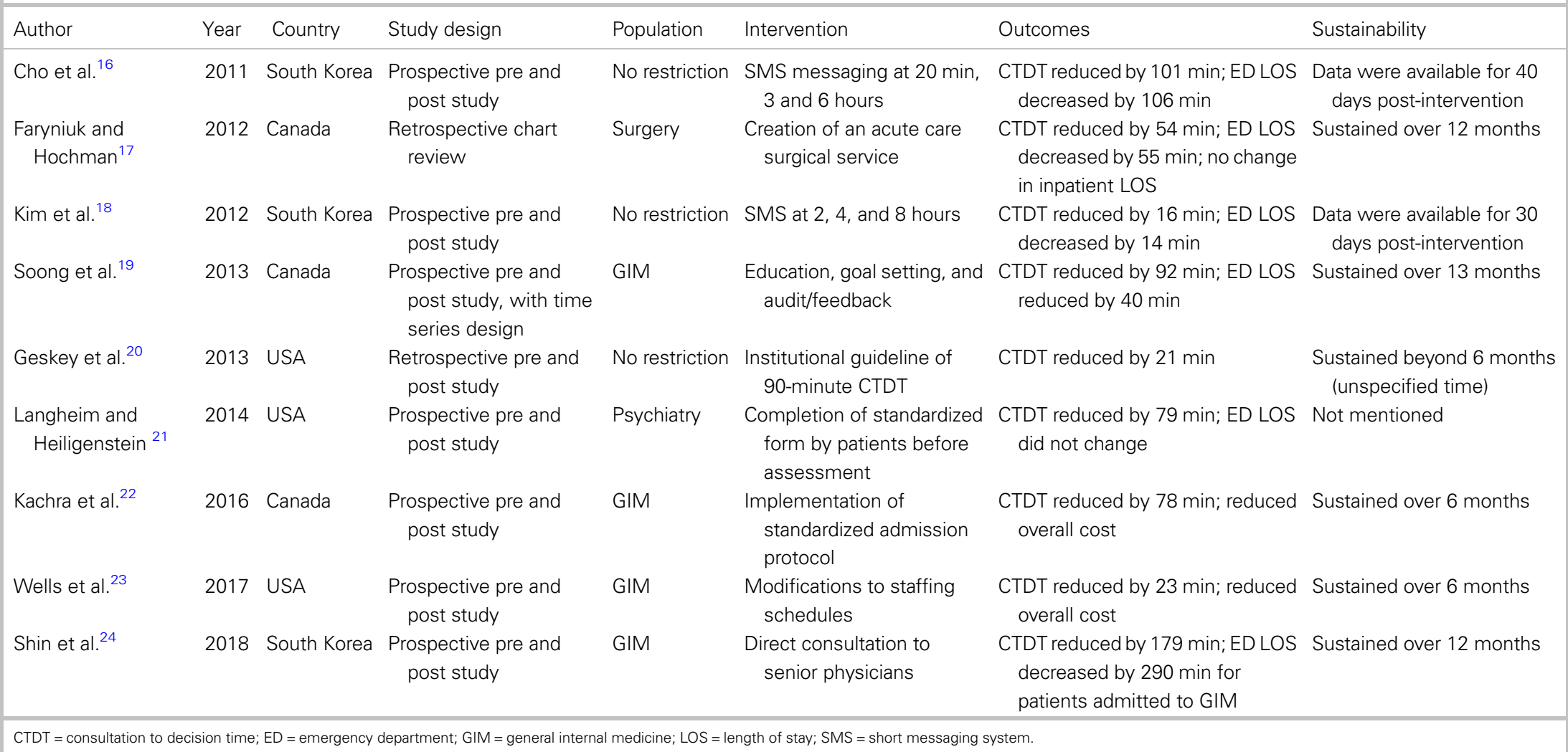


was 30 days post-intervention. This study was also rated as fair due to an insufficient assessment of balancing measures and short duration of follow-up.

\section{Standardization of the admission process}

In the study by Langheim and Heiligenstein, ${ }^{21}$ a standardized form was given to all ED patients requiring firsttime psychiatric consultations to improve efficiency. The standardized form included questions on the Diagnostic and Statistical Manual of Mental Disorders diagnosis, treatment history, past and present medications, among others. This study included only 37 patients pre-intervention and 9 post-intervention. The small sample size made it difficult to assess the improvement in outcomes using statistical measures. Data collection was inconsistent as some time points were inputted manually and others electronically. Consultation to decision time was reduced by 79 minutes in this study, but the ED length of stay did not change. The psychiatry residents expressed improved satisfaction after implementation of intervention. The study quality was rated as poor given the unclear sustainability, very small sample size, unclear uptake of the intervention, and inconsistent data collection.

Kachra et al. introduced a standardized admission protocol to the general internal medicine service. ${ }^{22}$ This protocol encouraged senior residents to make a disposition decision and enter admission orders within 2 hours of receiving a consultation request, without waiting for a junior resident to first complete the assessment. A reminder about utilization of the standardized protocol and summary data on consultation to decision time was emailed every 6 weeks. Surveys on barriers to admission were also collected. Consultation to decision time was reduced by 78 minutes, and cost per admission was lowered by $\$ 36$ (CAD). The outcomes were sustained over 6 months. Feedback forms identified high consultation volume, unclear patient disposition, and unstable patient status as barriers to admission. The study quality was rated as poor due to the lack of process and balancing measures, unclear uptake of intervention, small sample size, and lack of utilization of statistical measures.

\section{Restructuring the consultation process}

In the study by Shin et al., ED physicians contacted chief residents, junior attending, or attending physicians directly for consultation. ${ }^{23}$ After a disposition decision was made after a phone discussion, a junior resident was then asked to facilitate the admission process. Consultation to decision time was reduced by 179 minutes and ED length of stay by 290 minutes for patients admitted to general internal medicine. The results were sustained over 12 months. Admission order to ED departure time was reduced by 119 minutes, and inpatient mortality was unchanged. Run charts were used for statistical analyses. This study was rated as fair due to insufficient assessment of balancing measures, including provider experience and impact on resident education.

\section{Education, audit, and feedback}

In the study by Soong et al., education sessions on effective consultation and triage were held every 2 months for medicine residents to emphasize the potential adverse impact of prolonged ED wait times on patient care, and scenarios were presented to review principles of rapid triage. ${ }^{19}$ Personal consultation to decision time data for each resident were compiled and fed back every 2 weeks. A guideline of consultation to decision time of less than 1 hour was instituted. Consultation to decision time decreased by 92 minutes and ED length of stay by 40 minutes post-intervention. The data were presented using statistical process control charts and showed special cause variation. Process measures, including time to ED physician assessment and time to consultation request, did not change, but time to discharge from ED for admitted patients increased. There was no change in general internal medicine inpatient mortality or length of stay. The study quality was rated as good.

\section{Implementation of guidelines}

Geskey et al. established an institutional guideline, where the goal of physician arrival was within 30 minutes of consultation and that of reaching a disposition decision was within an additional 60 minutes. ${ }^{20}$ This was followed by weekly reports on referral and admission volume and consultation to decision time that were sent to hospital administrators, department chairs, and project leaders. Consultation to decision time decreased by 21 minutes, and this was sustained beyond 6 months. Time to discharge from the ED after submission of admission order was decreased by 18 minutes, but inpatient discharge time was delayed by 50 minutes. Run charts 
were used for data presentation. The study quality was rated as fair given other interventions were happening concurrently, as the largest consultation service made coverage modifications for ED referrals.

\section{Modification of staffing patterns}

Faryniuk and Hochman ${ }^{17}$ assessed the effectiveness of assigning a dedicated surgeon to provide daytime coverage for non-trauma surgical patients in reducing ED overcrowding. Data were obtained using retrospective chart review, and surgical patients with cholecystitis, appendicitis, or small bowel obstruction were included. Consultation to decision time was reduced by 54 minutes and ED length of stay decreased by 55 minutes. The results were sustained over 12 months. Process measures decreased, including time from consultation request to provider response, and time from provider response to admission decision was unchanged. The study quality was rated as poor given its small sample size (only 137 patients were identified 1 year following intervention), lack of balancing measures, and limited generalizability.

In the study by Wells et al., modifications to the ED consultation coverage by general internal medicine were done over three plan-do-study-act cycles. ${ }^{24}$ Continuous improvement in consultation to decision time was noted throughout these cycles, which ultimately resulted in the extension of ED coverage by existing services and the addition of a physician and an advanced practice registered nurse staffed shift. These interventions resulted in a reduction of 23 minutes in consultation to decision time and reduced overall cost, sustained over 6 months. The increase in cost of hiring of additional providers was offset by the reduction in cost per discharged patient (\$180 USD). Statistical process control charts were used to illustrate the improvement process. The study quality was rated as fair given the lack of mention of either process or balancing measures.

\section{DISCUSSION}

All studies reported improvement in consultation to decision time, and most studies also found a reduction in ED length of stay. Two studies reported on cost savings by reducing the consultation to decision time. ${ }^{22,23}$

Interventions with the highest effectiveness judging by the magnitude of consultation to decision time achieved include SMS messaging, ${ }^{16,18}$ direct consultation to senior physicians, ${ }^{24}$ standardization of the admission protocol, ${ }^{21,22}$ and education with audit and feedback. ${ }^{19}$

Among these, education with audit and feedback was the most rigorously studied but also required the greatest amount of resources, including regular education sessions and feedback for trainees. It was difficult to separate the effect of education from weekly audit and feedback in the study by Soong et al,, because they were implemented together. ${ }^{19}$ Other studies have shown that education, especially in the didactic form, is generally an ineffective strategy for eliciting behavioural changes. ${ }^{25,26}$ Therefore, it is possible that the individualized feedback to residents on their performance in this study was particularly important for improving efficiency.

Similarly, audit and feedback after implementation of an institutional guideline, resulted in a modest improvement in consultation to decision time in the study by Geskey et al. ${ }^{20}$ This all seem to support the efficacy of feedback in altering behaviours and outcomes in healthcare. ${ }^{27}$

In terms of feasibility, SMS messaging, direct consultation to senior physicians, and standardizing the admission protocol are all relatively easy to implement with lower resource requirement. In addition, standardization is a tenant of the $5 \mathrm{~S}$ model (sort, set in order, shine, standardize, and sustain) for quality improvement, and has resulted in improvement in efficiency in other projects in healthcare. ${ }^{28,29}$ However, further assessment of impact on provider experience is needed before consideration of a wider application.

Few studies explored changing staff scheduling to improve coverage of peak consultation times, likely because it represents one of the most resource-intensive interventions. In the study by Faryniuk and Hochman, no assessment of cost-effectiveness was reported. ${ }^{17}$ In the studies by Wells et al., the authors were able to carry out their interventions because of significant buy-in from key stakeholders in a smaller community hospital setting, and funding was available to add additional physician and registered nurse staffed shifts. ${ }^{23}$ Conducting similar interventions in larger centres might prove more challenging due to a larger number of personnel involved.

The relative effectiveness and feasibility of all interventions was summarized in Figure 2.

There are several limitations to this systematic review. Firstly, due to the significant heterogeneity amongst the interventions carried out, it was not possible to pool the outcome measures. In addition to the effectiveness of 

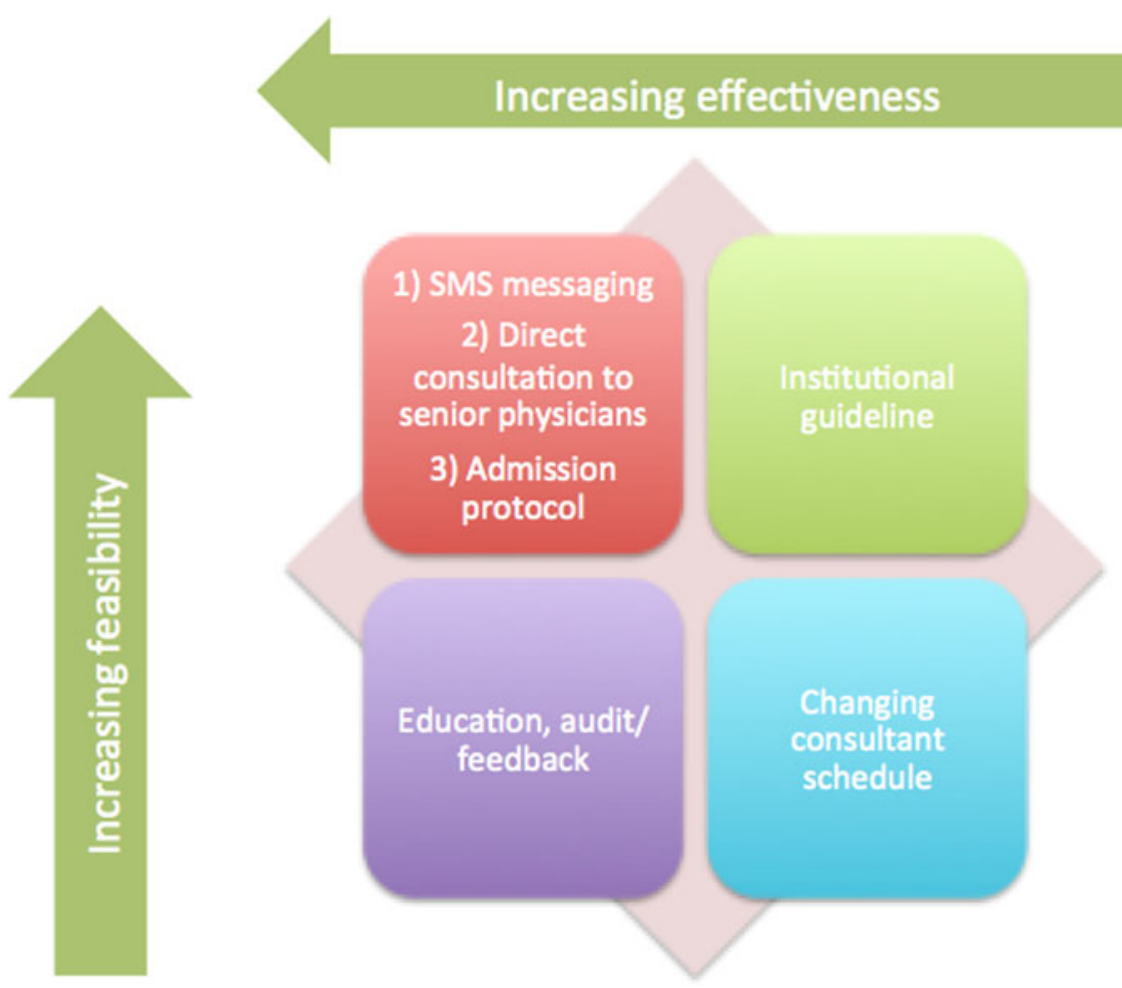

Figure 2. Grouping of interventions by effectiveness and feasibility in reducing CTDT

any given intervention, the extent to which consultation to decision time is reduced is potentially influenced by multiple other factors that may vary between institutions. Therefore, it is difficult to compare the relative efficacies of the various interventions studied. Secondly, only articles published in English were included, potentially missing important findings from studies conducted in non-English speaking countries. In addition, only the adult population was included in the literature search, so studies on reduction of consultation to decision time in pediatric populations would have been missed. Further, studies published as only abstracts were excluded as well, because it was difficult to extract all data points of interest from abstracts alone. Thirdly, all studies took place in North America and South Korea, potentially limiting its generalizability to countries with different healthcare systems. Fourthly, although the studies suggested improvement in consultation to decision time, it is unclear whether the interventions might have resulted in distress and anxiety for providers, especially for trainees who need to balance learning, patient safety, efficiency, and evaluations by supervisors. Soong et al. mentioned that some trainees expressed frustrations over being under greater pressure to complete consults quickly, but no formal assessment of provider experience was done in any of the studies included. ${ }^{19}$ Lastly, the impact of decreased consultation to decision time on patient safety outcomes, such as morbidity and mortality, was not well explored in any of the studies.

\section{CONCLUSIONS}

In summary, audit and feedback interventions in the form of SMS messaging, direct consultation to senior physicians, and standardization of the admission process appear to be the most feasible and effective interventions in reducing consultation to decision time and ED length of stay. The quality of published studies on strategies to reduce consultation to decision time was overall fair to poor, largely due to lack of assessment of process and balancing measures. Future quality improvement initiatives should take this into consideration in order to improve the applicability of future interventions.

Supplementary material: The supplementary material for this article can be found at https://doi.org/10.1017/cem.2019.435.

Acknowledgements: We wish to thank Alexandra (Sascha) Davis for her help with the literature search, and Hanna Kuk and Kylie $\mathrm{McNeil}$ for their input in editing the manuscript. 
Competing interests: None declared.

\section{REFERENCES}

1. Richardson DB. Increase in patient mortality at 10 days associated with emergency department overcrowding. $M 7 A$ 2006;184:213-6.

2. Bernstein SL, Aronsky D, Duseja R, et al.; Society for Academic Emergency Medicine, Emergency Department Crowding Task Force. The effect of emergency department crowding on clinically oriented outcomes. Acad Emerg Med 2009;16(1):1-10.

3. Krochmal P, Riley TA. Increased health care costs associated with ED overcrowding. Am 7 Emerg Med 1994;12(3):265-6.

4. Yancer DA, Foshee D, Cole H, et al. Managing capacity to reduce emergency department overcrowding and ambulance diversions. Ft Comm f Qual Patient Saf 2006;32(5):239-45.

5. Brouns SH, Stassen PM, Lambooij SL, et al. Organisational factors induce prolonged emergency department length of stay in elderly patients - a retrospective cohort study. PLoS One 2015; 10(8): e0135066.

6. Erenler AK, Akbulut S, Guzel M, et al. Reasons for overcrowding in the emergency department: experiences and suggestions of an education and research hospital. Turk 7 Emerg Med 2016;14(2):59-63.

7. Wilbert CC. Timeliness of care in the emergency department. QRB Qual Rev Bull 1984;10(4):99-108.

8. Lee PA, Rowe BH, Innes G, et al. Assessment of consultation impact on emergency department operations through novel metrics of responsiveness and decision-making efficiency. C7EM 2014;16(3):185-92.

9. Yoon P, Steiner I, Reinhardt G. Analysis of factors influencing length of stay in the emergency department. CFEM 2003;5(3):155-61.

10. Woods RA, Lee R, Ospina MB, et al. Consultation outcomes in the emergency department: exploring rates and complexity. CFEM 2008;10(1):25-31.

11. Lee RS, Woods R, Bullard M, Holroyd BR, Rowe BH. Consultations in the emergency department: a systematic review of the literature. Emerg Med $\mathcal{F}$ 2008;25(1):4-9.

12. Moher D, Liberati A, Tetzlaff J, Altman DG, The PRISMA Group. Preferred Reporting Items for Systematic Reviews and Meta-Analyses: The PRISMA statement. PLoS Med 2009;6(7):e1000097.

13. National Heart, Lung, and Blood Institute. NIH. Quality assessment tool for before-after (pre-post) studies with no control group; n. d. Available at: https://www.nhlbi.nih.gov/ health-topics/study-quality-assessment-tools (accessed May 01, 2018).

14. Gotlib Conn L, Nathens AB, Perrier L, et al. Quality of reporting on guideline, protocol, or algorithm implementation in adult trauma centres: a systematic review. Ann Surg 2019; epub.

15. Howell V, Schwartz AE, O'Leary JD, Mc Donnell C. The effect of the SQUIRE (Standards of QUality Improvement Reporting Excellence) guidelines on reporting standards in the quality improvement literature: a before-and-after study. BMF Qual Saf 2015;24(6):400-6.

16. Cho SJ, Jeong J, Han S, et al. Decreased emergency department length of stay by application of a computerized consultation management system. Acad Emerg Med 2011;18(4):398402.

17. Faryniuk AM, Hochman DJ. Effect of an acute care surgical service on the timeliness of care. Can 7 Surg 2013;56 (3):187-91.

18. Kim MJ, Park JM, Je SM, et al. Effects of a short text message reminder system on emergency department length of stay. Int 7 Med Inform 2012;81(5):296-302.

19. Soong C, High S, Morgan MW, Ovens H. A novel approach to improving emergency department consultant response times. BM7 Qual Saf 2013;22(4):299-305.

20. Geskey JM, Geeting G, West C, Hollenbeak CS. Improved physician consult response times in an academic emergency department after implementation of an institutional guideline. 7 Emerg Med 2013;44(5):999-1006.

21. Langheim FJ, Heiligenstein E. Evaluation of the timeliness of psychiatric consultations. 7 Clin Med Res 2014;6(4):242-4.

22. Kachra R, Walzak A, Hall S, et al. Resident-driven quality improvement pre-post intervention targeting reduction of emergency department decision to admit time. Can 7 GIM 2016;11(2):14-20.

23. Wells M, Coates E, Williams B, Blackmore C. Restructuring hospitalist work schedules to improve care timeliness and efficiency. BM7 Open Qual 2017;6(2):e000028.

24. Shin S, Lee SH, Kim DH, et al. The impact of the improvement in internal medicine consultation process on ED length of stay. Am 7 Emerg Med 2018;36(4):620-4.

25. Bloom B. Effects of continuing medical education on improving physician clinical care and patient health: a review of systematic reviews. Int 7 Technol Assess Health Care 2005;21:380-5.

26. Davis DA, Thomson MA, Oxman AD, Haynes RB. Changing physician performance. $\mathcal{7} A M A$ 1995;274:700-5.

27. Ivers N, Jamtvedt G, Flottorp S, et al. Audit and feedback: effects on professional practice and healthcare outcomes. Cochrane Database Syst Rev 2012;7:1-229.

28. Kruskal JB, Reedy A, Pascal L, Rosen MP, Boiselle PM. Quality initiatives: lean approach to improving performance and efficiency in a radiology department. Radiographics 2012;32(2):573-87.

29. Allaudeen N, Vashi A, Breckenridge JS, et al. Using lean management to reduce emergency department length of stay for medicine admissions. Qual Manag Healtb Care 2017;26(2):91-6. 